\title{
Inverse source problem in an advection-dispersion- reaction system: application to water pollution
}

\author{
Abdellatif El Badia and Adel Hamdi \\ Université de Technologie de Compiègne, Laboratoire de Mathématiques Appliquées de \\ Compiégne, BP 20529, 60205 Compiègne Cedex, France \\ E-mail: abdellatif.elbadia@utc.fr
}

Received 19 February 2007, in final form 6 June 2007

Published 11 September 2007

Online at stacks.iop.org/IP/23/2103

\begin{abstract}
In this paper, we consider the problem of identifying an unknown source $F(x, t)=\lambda(t) \delta(x-S)$ in the following system:

$\left(\partial_{t}-D \partial_{x x}+V \partial_{x}+R\right) u(x, t)=F(x, t), \quad 0<x<\ell, \quad 0<t<T$

$\left(\partial_{t}-D \partial_{x x}+V \partial_{x}+R\right) v(x, t)=R u(x, t), \quad 0<x<\ell, \quad 0<t<T$

from measured data $\left[\left\{v(a, t), \partial_{x} v(a, t)\right\},\left\{v(b, t), \partial_{x} v(b, t)\right\}\right]$ for appropriate points $a$ and $b$. Assuming that the source $F$ became inactive after the time $T^{*}$ (i.e. $\lambda(t)=0$ for $t \geqslant T^{*}$ ), we prove an identifiability result and propose an identification method.
\end{abstract}

(Some figures in this article are in colour only in the electronic version)

\section{Introduction}

Inverse source problems are important in many sectors of engineering. Among these, we quote two for which there is an extensive literature: identification of environment pollution sources and current dipolar sources in the so-called inverse electroencephalography/ magnetoencephalography problems (see $[1-6,8]$ and the references cited therein).

In this paper, we consider the problem of determining a source $F(x, t)=\lambda(t) \delta(x-S)$ in the following advection-dispersion-reaction system:

$$
\begin{aligned}
& \left(\partial_{t}-D \partial_{x x}+V \partial_{x}+R\right) u(x, t)=F(x, t), \quad 0<x<\ell, \quad 0<t<T \\
& \left(\partial_{t}-D \partial_{x x}+V \partial_{x}+R\right) v(x, t)=R u(x, t), \quad 0<x<\ell, \quad 0<t<T
\end{aligned}
$$

where $D, V, R$ are positive numbers, $S \in] 0, \ell\left[\right.$ and $\lambda \in L^{2}(0, T)$.

The main application (but not only the one) of our study is the identification of the source pollution $F$ of biological oxygen demand (BOD) in a river, from concentration measures of dissolved oxygen (DO) at appropriate points. Here, the concentrations of BOD and DO are, respectively, denoted by $u$ and $v$. 
As usual, to the evolution equations (1.1) one has to append initial and boundary conditions. For the first one, there is no restriction to start the time interval at some moment where no pollution has yet occurred. For the second one, physical considerations indicate that things are different at the two extreme points of the observed portion of the river, denoted by $(0, \ell)$. Indeed, in most situations of interest, transport is unidirectional in nature. It means that there is no significant transport upstream. Therefore, the null concentration at some point situated upstream can be used as the boundary condition. On the other hand, there are two options for modeling the downstream boundary: a zero gradient or a zero concentration assumption. The first option corresponds better to the transport physics; however if the downstream point is far enough from the source, the second one seems reasonable as well. To simplify the presentation, we will consider here only the first option. That corresponds to the following initial-boundary conditions, with initial-boundary conditions:

$$
\begin{array}{ll}
u(x, 0)=v(x, 0)=0 & \text { for } 0<x<\ell \\
u(0, t)=v(0, t)=0 & \text { for } 0<t<T \\
\partial_{x} u(\ell, t)=\partial_{x} v(\ell, t)=0 & \text { for } 0<t<T .
\end{array}
$$

It is well known (see [9]) that the problem (1.1), (1.2) has a unique solution, denoted here by $(u=u(x, t ; F), v=v(x, t ; F))$ where $u$ belongs to the functional space

$$
L^{2}\left(0, T ; H^{1}(0, \ell)\right) \cap C\left([0, T] ; L^{2}(0, \ell)\right)
$$

and

$$
v \in C\left([0, T] ; H^{2}(0, \ell)\right) .
$$

Thus, by the imbedding Sobolev theorem, for $0<a<b<\ell$, one can define the observation operator

$$
B[F]:=\left\{v(a, t ; F), \partial_{x} v(a, t ; F), v(b, t ; F), \partial_{x} v(b, t ; F), 0<t<T\right\} .
$$

That is the so-called direct problem. The inverse problem with which we are concerned here is the following.

(ISP) Given the records $\left\{d_{1}(t), d_{2}(t), d_{3}(t), d_{4}(t), 0<t<T\right\}$ of the concentration $v$ and $\partial_{x} v$ at two observation points $a, b \in(0, \ell)$, find the source $F$ such that

$$
B[F]=\left\{d_{1}(t), d_{2}(t), d_{3}(t), d_{4}(t), 0<t<T\right\} .
$$

The above inverse problem (ISP) is different to that considered in our previous study [3], although we are in both cases interested in the identification of source pollution in a river. Indeed, in [3] only the first equation in (1.1) has been considered where the measurements are taken directly on the concentration $u$. However, in our present study we consider the coupled equations given by (1.1), (1.2) where the measurements are made on the concentration $v$ which completely changes the nature of the problem. In our two studies, we aim to determine the location of the BOD source and to recover its intensity function from some concentration measurements. The principal advantage of using measurements on $v$ is the reduction of the long time required in [3] (5 days; see [7, 10]) in order to get the identification results. Nevertheless, the numerical results, carried out on the same example, show that those obtained by the first method are more accurate than those obtained by the second one, which was foreseeable. The organization of this paper is as follows. The main theorem on the uniqueness in our inverse problem is firstly presented in section 2 followed by some new lemmas. Section 3 is devoted to a numerical algorithm. Some numerical experiments are given in section 4. 


\section{Main theorem}

As stated in (ISP), the measurements of DO are made at two interior points. We also assume that, from some a priori knowledge of the localization of the source, one of the two points is chosen upstream and the other downstream with respect to the source, that is $0<a<S<b<\ell$. The main result (theorem 1) as in [3] also appeals to the concept of strategic point that we recall here for convenience of the reader.

Definition 1. Let $\left\{\psi_{n}\right\}$ be a complete orthonormal family of continuous functions in $L^{2}(0, \ell)$. Then a point $x_{0} \in(0, \ell)$ is said to be a 'strategic point' relative to the family $\left\{\psi_{n}\right\}$ if

$$
\psi_{n}\left(x_{0}\right) \neq 0 \quad \forall n .
$$

In particular, let $\left\{\psi_{n}\right\}$ denote the complete orthonormal family of eigenfunctions for the following Sturm-Liouville problem, in which the parameters, $D, V, R$ are those from the operator

$$
\begin{aligned}
& L:=\partial_{t}-D \partial_{x x}+V \partial_{x}+R \\
& -D \psi^{\prime \prime}(x)+\varrho \psi(x)=\mu \psi(x), \quad 0<x<\ell \\
& \psi(0)=\psi^{\prime}(\ell)-\alpha \psi(\ell)=0,
\end{aligned}
$$

where

$$
\alpha=\frac{-V}{2 D} \quad \text { and } \quad \varrho=\alpha^{2} D+R .
$$

One can easily verify that $\psi_{n}(x)=c_{n} \sin \left(\beta_{n} x\right)$ where $\left(\beta_{n}\right), n \geqslant 0$ is solution to $\beta \cot (\beta \ell)=\alpha$ listed in increasing order and $c_{n}$ is a normalization coefficient. The associated eigenvalues $\mu_{n}$ to $\psi_{n}$ are

$$
\mu_{n}=\varrho+D \beta_{n}^{2}
$$

Thus,

$\beta_{n}=(2 n+1) \frac{\pi}{2}-\epsilon_{n} \quad$ with $\quad 0<\epsilon_{n}<\pi / 2 \quad$ and $\quad \lim _{n \rightarrow \infty} \epsilon_{n}=0$,

so that

$$
\varrho<\mu_{n}<\mu_{n+1}, \mu_{n} \sim \frac{D^{2}}{\ell^{2}} \pi^{2} n^{2} \text { at infinity. }
$$

\subsection{Main theorem}

Theorem 1. Suppose $F_{j}(x, t)=\lambda_{j}(t) \delta\left(x-S_{j}\right)$ where $\lambda_{j} \in L^{2}(0, T)$ is such that for $j=1,2, \lambda_{j}(t) \geqslant 0$ with $\lambda_{j}(t)=0$ for $T^{*}<t<T$, and $S_{j} \in(a, b) j=1,2$.

If at least one of the points $a$ or $b$ is strategic with respect to the family $\left\{\psi_{n}\right\}$, then $B\left[F_{1}\right]=B\left[F_{2}\right]$ implies $S_{1}=S_{2}$ and $\lambda_{1}(t)=\lambda_{2}(t)$ a.e. in $(0, T)$.

\subsection{Some preliminary results}

Let $\left(u_{j}, v_{j}\right), j=1,2$ be the solutions to (1.1), (1.2). We define the differences $w=u_{2}-u_{1}$ and $z=v_{2}-v_{1}$ and obtain 


$$
\begin{array}{ll}
L[w](x, t)=\lambda_{2}(t) \delta\left(x-S_{2}\right)-\lambda_{1}(t) \delta\left(x-S_{1}\right), & 0<x<\ell, \quad 0<t<T, \\
L[z](x, t)=R w(x, t), & 0<x<\ell, \quad 0<t<T, \\
w(0, t)=z(0, t)=0, & 0<t<T, \\
\partial_{x} w(\ell, t)=\partial_{x} z(\ell, t)=0, & 0<t<T, \\
w(x, 0)=z(x, 0)=0 & 0<x<\ell,
\end{array}
$$

while $B\left[F_{1}\right]=B\left[F_{2}\right]$ means that

$$
z(a, t)=\partial_{x} z(a, t)=0 \quad \text { for } \quad 0<t<T
$$

and

$$
z(b, t)=\partial_{x} z(b, t)=0 \quad \text { for } \quad 0<t<T .
$$

Henceforth, throughout this paper, we choose $b$ as a strategic point relative to the family $\left\{\psi_{n}\right\}$. Furthermore, $r_{i}, i=1,2$, denote the solutions to the characteristic equation

$$
D r^{2}+V r-R=0
$$

which are given explicitly by $r_{1}=\frac{-V-\sqrt{V^{2}+4 R D}}{2 D}$ and $r_{2}=\frac{-V+\sqrt{V^{2}+4 R D}}{2 D}$.

Lemma 1 (observability). Let $T^{*}<T$. Let $(w, z)$ be the solution to (2.4). Then

$$
z(b, t)=0, \forall t \in] T^{*}, T\left[\Longrightarrow w\left(., T^{*}\right)=0 \quad \text { and } \quad z\left(., T^{*}\right)=0 \quad \text { in } \quad L^{2}(0, \ell)\right. \text {. }
$$

Proof. Since $\lambda(t)=0$ for $T^{*}<t<T$, the functions $w$ and $z$ satisfy

$$
\begin{array}{ll}
L[w](x, t)=0, & 0<x<\ell, \quad T^{*}<t<T, \\
L[z](x, t)=R w(x, t), & 0<x<\ell, \quad T^{*}<t<T, \\
w(x, 0)=z(x, 0)=0 & 0<x<\ell, \\
w(0, t)=z(0, t)=0, & T^{*}<t<T, \\
\partial_{x} w(\ell, t)=\partial_{x} z(\ell, t)=0, & T^{*}<t<T .
\end{array}
$$

Let now

$$
y_{w}(x, t)=\mathrm{e}^{\alpha x} w(x, t) \quad \text { and } \quad y_{z}(x, t)=\mathrm{e}^{\alpha x} z(x, t) \quad \text { with } \quad \alpha \text { given in (2.2). }
$$

Then, $w$ and $z$ are solutions to (2.7) if and only if $y_{w}$ and $y_{z}$ are, respectively, solutions of the following heat equations:

$$
\begin{array}{ll}
\partial_{t} y_{w}-D \partial_{x x} y_{w}+\varrho y_{w}=0, & 0<x<\ell, \quad T^{*}<t<T \\
y_{w}(0, t)=\partial_{x} y_{w}(\ell, t)-\alpha y_{w}(\ell, t)=0, & T^{*}<t<T, \\
y_{w}\left(., T^{*}\right)=\mathrm{e}^{\alpha x} w\left(., T^{*}\right), & 0<x<\ell,
\end{array}
$$

and

$$
\begin{array}{ll}
\partial_{t} y_{z}-D \partial_{x x} y_{z}+\varrho y_{z}=R \mathrm{e}^{\alpha x} w, & 0<x<\ell, \quad T^{*}<t<T \\
y_{z}(0, t)=\partial_{x} y_{z}(\ell, t)-\alpha y_{z}(\ell, t)=0, & T^{*}<t<T, \\
y_{z}\left(., T^{*}\right)=\mathrm{e}^{\alpha x} z\left(., T^{*}\right), & 0<x<\ell,
\end{array}
$$

which are given by the Fourier expansion

$$
y_{w}(x, t)=\sum_{n \geqslant 0} e_{n}(t) \psi_{n}(x) \quad \text { and } \quad y_{z}(x, t)=\sum_{n \geqslant 0} f_{n}(t) \psi_{n}(x)
$$


where $e_{n}$ and $f_{n}$ are, respectively, solutions to

$$
\begin{aligned}
& e_{n}^{\prime}(t)+\mu_{n} e_{n}(t)=0, \quad T^{*}<t<T \\
& e_{n}\left(T^{*}\right)=\left\langle y_{w}\left(., T^{*}\right), \psi_{n}\right\rangle_{L^{2}}
\end{aligned}
$$

and

$$
\begin{aligned}
& f_{n}^{\prime}(t)+\mu_{n} f_{n}(t)=R<\mathrm{e}^{\alpha x} w(., t), \quad \psi_{n}>_{L^{2}}, \quad T^{*}<t<T, \\
& f_{n}\left(T^{*}\right)=\left\langle y_{z}\left(., T^{*}\right), \psi_{n}\right\rangle_{L^{2}} .
\end{aligned}
$$

Here $\varrho$ is given in (2.2), $\left(\mu_{n}, \psi_{n}\right)$ denote the eigenvalues and eigenfunctions defined by (2.1).

Thus, one gets

$$
w(x, t)=\mathrm{e}^{-\alpha x} \sum_{n \geqslant 0}\left\langle y_{w}\left(., T^{*}\right), \psi_{n}\right\rangle_{L^{2}} \psi_{n}(x) \mathrm{e}^{-\mu_{n}\left(t-T^{*}\right)}
$$

and then

$$
\begin{aligned}
z(x, t)=\mathrm{e}^{-\alpha x} & \sum_{n \geqslant 0}\left\langle y_{z}\left(., T^{*}\right), \psi_{n}\right\rangle_{L^{2}} \mathrm{e}^{-\mu_{n}\left(t-T^{*}\right)} \psi_{n}(x) \\
& +\mathrm{e}^{-\alpha x} R \sum_{n \geqslant 0} \psi_{n}(x) \int_{T^{*}}^{t}\left\langle\mathrm{e}^{\alpha x} w(., r), \psi_{n}\right\rangle_{L^{2}} \mathrm{e}^{-\mu_{n}(t-r)} \mathrm{d} r
\end{aligned}
$$

where $\langle f, g\rangle_{L^{2}}$ denotes the $L^{2}(0, \ell)$ inner product $\int_{0}^{\ell} f(x) g(x) \mathrm{d} x$.

Now, by substituting $w$ given by (2.8) into the above expression of $z$, we obtain

$$
z(x, t)=\sum_{n \geqslant 0} \gamma_{n}(x, t) \mathrm{e}^{-\mu_{n}\left(t-T^{*}\right)}
$$

where

$\gamma_{n}(x, t)=\mathrm{e}^{-\alpha x}\left\{R\left(t-T^{*}\right)\left\langle y_{w}\left(., T^{*}\right), \psi_{n}\right\rangle_{L^{2}}+\left\langle y_{z}\left(., T^{*}\right), \psi_{n}\right\rangle_{L^{2}}\right\} \psi_{n}(x)$.

Actually, from (2.3), one sees that the expansion on the RHS of (2.9) is uniformly convergent for all $t \geqslant t_{0}>T^{*}$ and represents a real analytic function in $] T^{*}, \infty[$ for every $x \in(0, \ell)$. That gives a sense to $z(b, t)$ and $w(b, t)$ for $t>T^{*}$.

Now, since

$$
z(b, t)=0, \quad \forall t \in] T^{*}, T[,
$$

by analytic continuation we conclude that

$$
\left.\sum_{n \geqslant 0} \gamma_{n}(b, t) \mathrm{e}^{-\mu_{n}\left(t-T^{*}\right)}=0, \quad \forall t \in\right] T^{*},+\infty[,
$$

where $\gamma_{n}$ is given by (2.10). Therefore, using again (2.3), one can successively deduce that all the coefficients of $\mathrm{e}^{-\mu_{n}\left(t-T^{*}\right)}$ in the series (2.11) are such that

$$
\lim _{t \rightarrow+\infty} \gamma_{n}(b, t)=0, \quad \forall n \in \mathbb{N} .
$$

Indeed, by rewriting (2.11) as

$$
\gamma_{0}(b, t)+\sum_{n \geqslant 1} \gamma_{n}(b, t) \mathrm{e}^{\left(\mu_{0}-\mu_{n}\right)\left(t-T^{*}\right)}=0,
$$

taking $t \longrightarrow \infty$, one obtains

$$
\lim _{t \rightarrow+\infty} \gamma_{0}(b, t)=0
$$


since the series

$$
\sum_{n \geqslant 1} \gamma_{n}(b, t) \mathrm{e}^{\left(\mu_{0}-\mu_{n}\right)\left(t-T^{*}\right)}
$$

converges uniformly on $\left[t_{0},+\infty\left[\right.\right.$, with $t_{0}>T_{*}$ and

$$
\lim _{t \longrightarrow+\infty} \gamma_{n}(b, t) \mathrm{e}^{\left(\mu_{0}-\mu_{n}\right)\left(t-T^{*}\right)}=0 .
$$

By repeating the argument for $n=1,2$, etc, one proves that

$$
\lim _{t \rightarrow+\infty} \gamma_{n}(b, t)=0, \quad \forall n \geqslant 1 .
$$

Now, since $b$ is strategic, one has from (2.10)

$\lim _{t \longrightarrow+\infty} R\left(t-T^{*}\right)\left\langle y_{w}\left(., T^{*}\right), \psi_{n}\right\rangle_{L^{2}}+\left\langle y_{z}\left(., T^{*}\right), \psi_{n}\right\rangle_{L^{2}}=0, \quad \forall n \in \mathbb{N}$.

Therefore,

$$
\left\langle y_{w}\left(., T^{*}\right), \psi_{n}\right\rangle_{L^{2}}=0 \quad \text { and then } \quad\left\langle y_{z}\left(., T^{*}\right), \psi_{n}\right\rangle_{L^{2}}=0, \quad \forall n \in \mathbb{N}
$$

which means

$$
y_{w}\left(., T^{*}\right)=y_{z}\left(., T^{*}\right)=0 \quad \text { in } \quad L^{2}(0, \ell)
$$

and consequently,

$$
w\left(., T^{*}\right)=z\left(., T^{*}\right)=0 \quad \text { in } \quad L^{2}(0, \ell) .
$$

This proves lemma 1.

Lemma 2. Let $(w, z)$ be the solution to the system (2.4), satisfying (2.5) and such that $z(b, t)=0, \forall t \in] 0, T[$. Then

$$
\int_{0}^{T^{*}} w(a, t) \mathrm{d} t=\int_{0}^{T^{*}} \partial_{x} w(a, t) \mathrm{d} t=0 .
$$

Proof. Since $a<S$, one has

$$
\begin{array}{ll}
L[w](x, t)=0, & 0<x<a, \quad 0<t<T^{*}, \\
L[z](x, t)=R w(x, t), & 0<t<T^{*}, \\
w(x, 0)=z(x, 0)=0, & 0<x<a, \\
w(0, t)=z(0, t)=0, & 0<t<T^{*} .
\end{array}
$$

Let now $f_{0}$ be the solution of the differential equation

$$
\begin{aligned}
& \left.D f_{0}^{\prime \prime}(x)+V f_{0}^{\prime}(x)-R f_{0}(x)=\theta_{0}(x) \text { in }\right] 0, a[ \\
& f_{0}(0)=f_{0}(a)=0,
\end{aligned}
$$

where $\theta_{0}=\theta_{1}-\theta_{2}$.

Multiplying respectively the first equation of (2.12) by $f_{0}$, the second equation by $\theta_{0}$ and integrating with respect to $x$ and $t$ over $] 0, a[\times] 0, T^{*}[$, one has

$$
\begin{aligned}
& \int_{0}^{a} f_{0}(x) w\left(x, T^{*}\right) \mathrm{d} x+\int_{0}^{T^{*}}\left[-D \partial_{x} w f_{0}+D w f_{0}^{\prime}+V w f_{0}\right]_{0}^{a} \mathrm{~d} t=\int_{0}^{T^{*}} \int_{0}^{a} w(x, t) \theta_{0}(x) \mathrm{d} x \mathrm{~d} t \\
& \text { and } \\
& \int_{0}^{a} \theta_{0}(x) z\left(x, T^{*}\right) \mathrm{d} x+\int_{0}^{T^{*}}\left[-D \partial_{x} z \theta_{0}+D z \theta_{0}^{\prime}+V z \theta_{0}\right]_{0}^{a} \mathrm{~d} t=R \int_{0}^{T^{*}} \int_{0}^{a} w(x, t) \theta_{0}(x) \mathrm{d} x \mathrm{~d} t .
\end{aligned}
$$


Here, $[z]_{a}^{b}$ denotes $z(b)-z(a)$.

Thus,

$$
\begin{gathered}
R D f_{0}^{\prime}(a) \int_{0}^{T^{*}} w(a, t) \mathrm{d} t=\int_{0}^{T^{*}}\left[\left\{D \theta_{0}^{\prime}(a)+V \theta_{0}(a)\right\} z(a, t)-D \theta_{0}(a) \partial_{x} z(a, t)\right] \mathrm{d} t \\
+\int_{0}^{a}\left[z\left(x, T^{*}\right) \theta_{0}(x)-R w\left(x, T^{*}\right) f_{0}(x)\right] \mathrm{d} x .
\end{gathered}
$$

In addition, multiplying the first equation of (2.12) by $\theta_{0}$ and integrating with respect $x$ and $t$ over $] 0, a[\times] 0, T^{*}[$, one gets

$D \theta_{0}(a) \int_{0}^{T^{*}} \partial_{x} w(a, t) \mathrm{d} t=\int_{0}^{a} w\left(x, T^{*}\right) \theta_{0}(x) \mathrm{d} x+\left\{D \theta_{0}^{\prime}(a)+V \theta_{0}(a)\right\} \int_{0}^{T^{*}} w(a, t) \mathrm{d} t$.

Moreover, $f_{0}^{\prime}(a) \neq 0$. To see that, it suffices to multiply (2.13) by $\theta_{0}$ and integrate by parts over $[0, a]$.

Now, since $\lambda_{j}(t)=0$ in $] T^{*}, T[$ and $z(b, t)=0$ in $] 0, T[$, one has from lemma 1 $w\left(., T^{*}\right)=0$ in $L^{2}(0, \ell)$. From this and according to (2.14), (2.15) one obtains

$$
\int_{0}^{T^{*}} w(a, t) \mathrm{d} t=0
$$

and then

$$
\int_{0}^{T^{*}} \partial_{x} w(a, t) \mathrm{d} t=0
$$

In addition, by the same method we get a similar result at the point $b$ which is given by the following lemma.

Lemma 3. Let $(w, z)$ be the solution to the system (2.4), satisfying (2.6). Then,

$$
\int_{0}^{T^{*}} w(b, t) \mathrm{d} t=\int_{0}^{T^{*}} \partial_{x} w(b, t) \mathrm{d} t=0 .
$$

Proof 3. Since $S<b$, one gets

$$
\begin{array}{ll}
L[w](x, t)=0 & b<x<\ell, \quad 0<t<T^{*}, \\
L[z](x, t)=R w(x, t) & b<x<\ell, \quad 0<t<T^{*}, \\
w(x, 0)=z(x, 0)=0 & b<x<\ell, \\
\partial_{x} w(\ell, t)=\partial_{x} z(\ell, t)=0 & 0<t<T^{*} .
\end{array}
$$

To prove lemma 3 , we proceed in several steps.

Step 1 . Let $\theta_{b}=\theta_{1}-\mathrm{e}^{\left(r_{1}-r_{2}\right) b} \theta_{2}$, which satisfies $\theta_{b}(b)=0$. For reader's convenience, we set

$$
I_{i}(x)=D \theta_{i}^{\prime}(x)+V \theta_{i}(x) \quad i=1,2, b .
$$

Multiplying the first equation of (2.16), by $\theta_{b}$, integrating with respect $x$ and $t$ over ]$b, \ell[\times] 0, T^{*}[$ and from lemma 1 , one gets

$$
I_{b}(\ell) \int_{0}^{T^{*}} w(\ell, t) \mathrm{d} t=D \theta_{b}^{\prime}(b) \int_{0}^{T^{*}} w(b, t) \mathrm{d} t .
$$


Step 2. Consider now the differential equation

$$
\begin{aligned}
& \left.D f^{\prime \prime}(x)+V f^{\prime}(x)-R f(x)=\theta(x) \text { in }\right] b, \ell[ \\
& f(b)=f(\ell)=0,
\end{aligned}
$$

and denote by $f_{b}$ and $f_{1}$ the solutions associated respectively to (2.18) with $\theta=\theta_{b}$ and $\theta=\theta_{1}$.

Then, multiplying the first equation of (2.16) by $f_{b}$, the second by $\theta_{b}$, integrating with respect $x$ and $t$ over $] b, \ell[\times] 0, T^{*}[$ and from lemma 1 and (2.6), one obtains, respectively,

$D f_{b}^{\prime}(\ell) \int_{0}^{T^{*}} w(\ell, t) \mathrm{d} t-D f_{b}^{\prime}(b) \int_{0}^{T^{*}} w(b, t) \mathrm{d} t=\int_{0}^{T^{*}} \int_{b}^{\ell} \theta_{b}(x) w(x, t) \mathrm{d} x \mathrm{~d} t$ and

$$
R I_{b}(\ell) \int_{0}^{T^{*}} z(\ell, t) \mathrm{d} t=\int_{0}^{T^{*}} \int_{b}^{\ell} \theta_{b}(x) w(x, t) \mathrm{d} x \mathrm{~d} t
$$

Thus,

$$
R D f_{b}^{\prime}(\ell) \int_{0}^{T^{*}} w(\ell, t) \mathrm{d} t-R D f_{b}^{\prime}(b) \int_{0}^{T^{*}} w(b, t) \mathrm{d} t=I_{b}(\ell) \int_{0}^{T^{*}} z(\ell, t) \mathrm{d} t .
$$

In a similar way as above, multiplying the first equation of (2.16) by $f_{1}$, the second by $\theta_{1}$, integrating with respect to $x$ and $t$ on $] b, \ell[\times] 0, T^{*}[$, one gets from lemma 1 and (2.6)

$$
R D f_{1}^{\prime}(\ell) \int_{0}^{T^{*}} w(\ell, t) \mathrm{d} t-R D f_{1}^{\prime}(b) \int_{0}^{T^{*}} w(b, t) \mathrm{d} t=I_{1}(\ell) \int_{0}^{T^{*}} z(\ell, t) \mathrm{d} t
$$

from which and (2.19) one deduces

$\left[I_{1}(\ell) f_{b}^{\prime}(\ell)-I_{b}(\ell) f_{1}^{\prime}(\ell)\right] \int_{0}^{T^{*}} w(\ell, t) \mathrm{d} t=\left[I_{1}(\ell) f_{b}^{\prime}(b)-I_{b}(\ell) f_{1}^{\prime}(b)\right] \int_{0}^{T^{*}} w(b, t) \mathrm{d} t$.

Step 4. Multiplying the first equation of (2.16), by $\theta_{1}$, integrating with respect to $x$ and $t$ over ]$b, \ell[\times] 0, T^{*}[$ once again from lemma 1 one gets

$D \theta_{1}(b) \int_{0}^{T^{*}} \partial_{x} w(b, t) \mathrm{d} t+I_{1}(\ell) \int_{0}^{T^{*}} w(\ell, t) \mathrm{d} t-I_{1}(b) \int_{0}^{T^{*}} w(b, t)=0$.

Step 5. To complete the proof of lemma 3, it suffices to consider (2.17), (2.20) and show that the quantity

$$
\triangle=I_{b}(b)\left[I_{1}(\ell) f_{b}^{\prime}(\ell)-I_{b}(\ell) f_{1}^{\prime}(\ell)\right]-I_{b}(\ell)\left[I_{1}(\ell) f_{b}^{\prime}(b)-I_{b}(\ell) f_{1}^{\prime}(b)\right]
$$

is not null. We therefore obtain

$$
\int_{0}^{T^{*}} w(b, t)=\int_{0}^{T^{*}} w(\ell, t)=0
$$

and then from $(2.21)$

$$
\int_{0}^{T^{*}} \partial_{x} w(b, t) \mathrm{d} t=0
$$

Indeed, at first, since

$$
r_{1}=\frac{-V-\sqrt{V^{2}+4 R D}}{2 D} \quad \text { and } \quad r_{2}=\frac{-V+\sqrt{V^{2}+4 R D}}{2 D},
$$

one can easily see that

$$
I_{1}(\ell)=\left(D r_{1}+V\right) \mathrm{e}^{r_{1} \ell}<0, \quad I_{2}(\ell)=\left(D r_{2}+V\right) \mathrm{e}^{r_{2} \ell}>0
$$


and then

$$
I_{b}(\ell)=I_{1}(\ell)-\mathrm{e}^{\left(r_{1}-r_{2}\right) b} I_{2}(\ell)<0 .
$$

Moreover, for $x>b$ one has

$$
\theta_{b}(x)=\theta_{1}(x)-\mathrm{e}^{\left(r_{1}-r_{2}\right) b} \theta_{2}(x)=\mathrm{e}^{r_{1}}\left[\mathrm{e}^{r_{1}(x-b)}-\mathrm{e}^{r_{2}(x-b)}\right]<0 .
$$

Thus,

$$
\theta=\theta_{1}-\frac{I_{1}(\ell)}{I_{b}(\ell)} \theta_{b}>0 \quad \text { in } \quad[b, \ell] .
$$

Furthermore, let $\sigma_{i}(x)=\mathrm{e}^{s_{i} x}$ be the solution to the differential equation

$$
-D \sigma^{\prime \prime}+V \sigma^{\prime}+R \sigma=0
$$

where $s_{1}=-r_{1}$ and $s_{2}=-r_{2}$.

Consider the function

$$
\sigma_{b}=\sigma_{1}-\mathrm{e}^{\left(s_{1}-s_{2}\right) b} \sigma_{2}
$$

which satisfies $\sigma_{b}(x)=\mathrm{e}^{s_{1} b} \mathrm{e}^{s_{1}(x-b)}-\mathrm{e}^{s_{2}(x-b)}>0$ for $x>b$ since $s_{1}>s_{2}$.

Now, if we multiply (2.18) with $\theta=\theta_{1}-\frac{I_{1}(\ell)}{I_{b}(\ell)} \theta_{b}$, respectively by $\sigma_{2}$ and $\sigma_{b}$ and integrate by parts over $[b, \ell]$, we get

$$
\left[f_{1}^{\prime}(\ell)-\frac{I_{1}(\ell)}{I_{b}(\ell)} f_{b}^{\prime}(\ell)\right] \mathrm{e}^{s_{2} \ell}-\left[f_{1}^{\prime}(b)-\frac{I_{1}(\ell)}{I_{b}(\ell)} f_{b}^{\prime}(b)\right] \mathrm{e}^{s_{2} b}=\int_{b}^{\ell} \theta(x) \sigma_{2}(x) \mathrm{d} x>0
$$

and

$$
\sigma_{b}(\ell)\left[f_{1}^{\prime}(\ell)-\frac{I_{1}(\ell)}{I_{b}(\ell)} f_{b}^{\prime}(\ell)\right]=\int_{b}^{\ell} \theta(x) \sigma_{b}(x) \mathrm{d} x>0 .
$$

On the other hand, one has

$$
\frac{I_{b}(b)}{I_{b}(\ell)}=\gamma \mathrm{e}^{s_{2}(\ell-b)}
$$

where

$$
\gamma=\frac{2 \sqrt{V^{2}+4 R D}}{\left.V+\sqrt{V^{2}+4 R D}+\left[\sqrt{V^{2}+4 R D}-V\right)\right] \mathrm{e}^{\left(r_{1}-r_{2}\right)(\ell-b)}}>1 .
$$

Therefore, from (2.22), (2.23), and (2.25), we obtain

$$
\begin{aligned}
{\left[f_{1}^{\prime}(b)-\frac{I_{1}(\ell)}{I_{b}(\ell)} f_{b}^{\prime}(b)\right] } & <\mathrm{e}^{s_{2}(\ell-b)}\left[f_{1}^{\prime}(\ell)-\frac{I_{1}(\ell)}{I_{b}(\ell)} f_{b}^{\prime}(\ell)\right] \\
& <\gamma \mathrm{e}^{s_{2}(\ell-b)}\left[f_{1}^{\prime}(\ell)-\frac{I_{1}(\ell)}{I_{b}(\ell)} f_{b}^{\prime}(\ell)\right]
\end{aligned}
$$

and from $(2.24)$

$$
f_{1}^{\prime}(b)-\frac{I_{1}(\ell)}{I_{b}(\ell)} f_{b}^{\prime}(b)-\left[f_{1}^{\prime}(\ell)-\frac{I_{1}(\ell)}{I_{b}(\ell)} f_{b}^{\prime}(\ell)\right] \frac{I_{b}(b)}{I_{b}(\ell)}<0 .
$$

Thus,

$$
\Delta=I_{b}^{2}(\ell)\left\{f_{1}^{\prime}(b)-\frac{I_{1}(\ell)}{I_{b}(\ell)} f_{b}^{\prime}(b)-\frac{I_{b}(b)}{I_{b}(\ell)}\left[f_{1}^{\prime}(\ell)-\frac{I_{1}(\ell)}{I_{b}(\ell)} f_{b}^{\prime}(\ell)\right]\right\}<0 .
$$

Lemma 4 (uniqueness). Let $S=S_{1}=S_{2}$ and $(w, z)$ be the solution to the system (2.4). If $z(b, t)=0, \forall t \in] 0, T\left[\right.$, then $\lambda=0$ in $L^{2}(0, T)$. 
Proof. Let us set $y_{w}(x, t)=\mathrm{e}^{\alpha x} w(x, t)$ and $y_{z}(x, t)=\mathrm{e}^{\alpha x} z(x, t)$ with $\alpha$ given in (2.2). Then, $(w, z)$ is solution of (2.4) with $S=S_{1}=S_{2}$ if and only if $\left(y_{w}, y_{z}\right)$ is solution of the following system:

$$
\begin{array}{lll}
\partial_{t} y_{w}-D \partial_{x x} y_{w}+\varrho y_{w}=\mathrm{e}^{\alpha S}\left[\lambda_{2}(t)-\lambda_{1}(t)\right] \delta(x-S), & 0<x<\ell, \quad 0<t<T^{*} \\
\partial_{t} y_{z}-D \partial_{x x} y_{z}+\varrho y_{z}=R \mathrm{e}^{\alpha x} w, & 0<x<\ell, \quad 0<t<T^{*} \\
y_{w}(0, t)=\partial_{x} y_{w}(\ell, t)-\alpha y_{w}(\ell, t)=0, & 0<t<T^{*} \\
y_{z}(0, t)=\partial_{x} y_{z}(\ell, t)-\alpha y_{z}(\ell, t)=0, & 0<t<T^{*} \\
y_{w}(x, 0)=y_{z}(x, 0)=0, & 0<x<\ell,
\end{array}
$$

which is given by the Fourier expansion

$$
y_{w}(x, t)=\sum_{n \geqslant 0} e_{n}(t) \psi_{n}(x) ; \quad y_{z}(x, t)=\sum_{n \geqslant 0} f_{n}(t) \psi_{n}(x)
$$

where $e_{n}$ and $f_{n}$ are, respectively, solution to

$$
\begin{aligned}
& e_{n}^{\prime}(t)+\mu_{n} e_{n}(t)=\mathrm{e}^{\alpha S}\left[\lambda_{2}(t)-\lambda_{1}(t)\right] \psi_{n}(S), \quad 0<t<T^{*} \\
& e_{n}(0)=0
\end{aligned}
$$

and

$$
\begin{aligned}
& f_{n}^{\prime}(t)+\mu_{n} f_{n}(t)=R<\mathrm{e}^{\alpha x} w(., t), \psi_{n}>_{L^{2}}, \quad 0<t<T^{*} \\
& f_{n}(0)=0 .
\end{aligned}
$$

Thus,

$$
w(x, t)=\mathrm{e}^{\alpha(S-x)} \sum_{n \geqslant 0} \psi_{n}(S) \psi_{n}(x) \int_{0}^{t}\left[\lambda_{2}(r)-\lambda_{1}(r)\right] \mathrm{e}^{-\mu_{n}(t-r)} \mathrm{d} r
$$

and

$$
z(x, t)=R \mathrm{e}^{-\alpha x} \sum_{n \geqslant 0} \psi_{n}(x) \int_{0}^{t}<\mathrm{e}^{\alpha x} w(., r), \psi_{n}>_{L^{2}} \mathrm{e}^{-\mu_{n}(t-r)} \mathrm{d} r .
$$

Now, by substituting (2.26) into (2.27) one gets

$$
z(x, t)=R \mathrm{e}^{\alpha(S-x)} \sum_{n \geqslant 0} \psi_{n}(x) \psi_{n}(S) \int_{0}^{t} \int_{0}^{r}\left[\lambda_{2}(\eta)-\lambda_{1}(\eta)\right] \mathrm{e}^{-\mu_{n}(t-\eta)} \mathrm{d} \eta \mathrm{d} r
$$

which can be rewritten as follows:

$$
z(x, t)=R \mathrm{e}^{\alpha(S-x)} \sum_{n \geqslant 0} \psi_{n}(x) \psi_{n}(S) \int_{0}^{t} \int_{\eta}^{t}\left[\lambda_{2}(\eta)-\lambda_{1}(\eta)\right] \mathrm{e}^{-\mu_{n}(t-\eta)} \mathrm{d} r \mathrm{~d} \eta
$$

which means

$$
z(x, t)=R \mathrm{e}^{\alpha(S-x)} \sum_{n \geqslant 0} \psi_{n}(x) \psi_{n}(S) \int_{0}^{t}\left[\lambda_{2}(\eta)-\lambda_{1}(\eta)\right](t-\eta) \mathrm{e}^{-\mu_{n}(t-\eta)} \mathrm{d} \eta .
$$

Thus,

$$
z(x, t)=\int_{0}^{t}\left[\lambda_{2}(\eta)-\lambda_{1}(\eta)\right] \Phi(x, t-\eta) \mathrm{d} \eta
$$

where

$$
\Phi(x, t-\eta)=R \mathrm{e}^{\alpha(S-x)} \sum_{n \geqslant 0} \psi_{n}(x) \psi_{n}(S)(t-\eta) \mathrm{e}^{-\mu_{n}(t-\eta)} .
$$


The above inversion of integration and summation is justified by Lebesgue's theorem of dominated convergence.

Now, since $z(b, t)=0$ for $0<t<T$, one gets

$$
\left.\int_{0}^{t}\left[\lambda_{2}(\eta)-\lambda_{1}(\eta)\right] \Phi(b, t-\eta) \mathrm{d} \eta=0, \quad \forall t \in\right] 0, T[.
$$

According to Titchmarsh's theorem on convolution of $L^{1}$ functions [12], the functions $\lambda$ and $\Phi$ must vanish identically at least in intervals ]0, $T^{\prime}[$ and $] 0, T^{\prime \prime}\left[\right.$, respectively, with $T^{\prime}$ and $T^{\prime \prime}$ such that $T^{\prime}+T^{\prime \prime} \geqslant T$.

Now, if $\Phi=0$ in $] 0, T^{\prime \prime}\left[\right.$ with $T^{\prime \prime}>0$. By analytic continuation, one has $\Phi=0$ in ] $0,+\infty\left[\right.$ and therefore $\psi_{n}(S) \psi_{n}(b)=0 \forall n$.

Since $b$ is strategic, we obtain $\psi_{n}(S)=0 \forall n$, which is impossible according to (2.3). Thus,

$$
\left.\lambda_{2}=\lambda_{1}, \quad \text { a.e. in }\right] 0, T[\text {. }
$$

\subsection{Proof of the main theorem}

Multiplying the first equation of (2.4) by $\theta_{i}=\mathrm{e}^{r_{i} x}, i=1,2$, integrating with respect to $x$ and $t$ over $] a, b[\times] 0, T^{*}[$ and, therefore, by using (2.5), (2.6) and from lemmas 1-3 we get

$$
\left\{\begin{array}{l}
\bar{\lambda}_{2} \mathrm{e}^{r_{1} S_{2}}=\bar{\lambda}_{1} \mathrm{e}^{r_{1} S_{1}} \\
\bar{\lambda}_{2} \mathrm{e}^{r_{2} S_{2}}=\bar{\lambda}_{1} \mathrm{e}^{r_{2} S_{1}}
\end{array}\right.
$$

where $\overline{\lambda_{i}}=\int_{0}^{T^{*}} \lambda_{i}(t) \mathrm{d} t, i=1,2$. Since $r_{1} \neq r_{2}$ and $\bar{\lambda}_{i}>0$, one has $S_{1}=S_{2}$ and then $\bar{\lambda}_{1}=\bar{\lambda}_{2}$.

Then from lemma 4 one has $\lambda_{1}=\lambda_{2}$ a.e. in $] 0, T[$. This ends the proof of the theorem.

\section{Identification}

Given the records data $d_{i}(t), i=1 \leqslant 4,0<t<T$, that is $\left\{v(a, t ; F), \partial_{x} v(a, t ; F)\right\}$, $\left\{v(b, t ; F), \partial_{x} v(b, t ; F)\right\}$, for $0<t<T$, we would like to determine the scalar $S$ and the function $\lambda(t)$. To that, the method we will present requires, based on the knowledge of, $u\left(x, T^{*}\right), v\left(x, T^{*}\right)$ for $0<x<\ell, \int_{0}^{T^{*}} u(a, t) \mathrm{d} t, \int_{0}^{T^{*}} u(b, t) \mathrm{d} t, \int_{0}^{T^{*}} \partial_{x} u(a, t) \mathrm{d} t$ and $\int_{0}^{T^{*}} \partial_{x} u(b, t) \mathrm{d} t$, the prior estimation of $u\left(x, T^{*}\right)$ and $v\left(x, T^{*}\right)$.

For the reader's convenience, we proceed in several steps.

Step 1 . The functions $u\left(x, T^{*}\right)=u_{T^{*}}$ and $v\left(x, T^{*}\right)=v_{T^{*}}$ are determined from data $d_{1}(t)$ and $d_{3}(t), T^{*}<t<T$ by using the system

$$
\begin{array}{ll}
L[u](x, t)=0, & 0<x<\ell, \quad T^{*}<t<T, \\
L[v](x, t)=R u(x, t), & 0<x<\ell, \quad T^{*}<t<T, \\
u(0, t)=v(0, t)=0, & T^{*}<t<T, \\
\partial_{x} u(\ell, t)=\partial_{x} v(\ell, t)=0, & T^{*}<t<T, \\
u\left(x, T^{*}\right)=u_{T^{*}}(x) & 0<x<\ell, \\
v\left(x, T^{*}\right)=v_{T^{*}}(x) & 0<x<\ell,
\end{array}
$$


where the right-hand side of the first equation is null because $\lambda(t)=0$ for $T^{*}<t<T$. It is a classical observability problem for which we use a least-squares regularized method.

Step 2. The data $d_{i}(t), i \leqslant i \leqslant 4,0<t<T^{*}$, are used in equations from section 2 to evaluate several integral quantities needed in the next step as follows. As identifiability techniques, we use the measurement data, respectively, $d_{1}(t), d_{2}(t)$ for $0<t<T^{*}$ and $d_{3}(t), d_{4}(t)$ for $0<t<T^{*}$ to compute, respectively,

$\int_{0}^{T^{*}} u(a, t) \mathrm{d} t, \int_{0}^{T^{*}} \partial_{x} u(a, t) \mathrm{d} t \quad$ and $\quad \int_{0}^{T^{*}} u(b, t) \mathrm{d} t, \int_{0}^{T^{*}} \partial_{x} u(b, t) \mathrm{d} t$.

Indeed,

$$
\int_{0}^{T^{*}} u(a, t) \mathrm{d} t \quad \text { and } \quad \int_{0}^{T^{*}} \partial_{x} u(a, t) \mathrm{d} t
$$

are obtained from formulae (2.14) and (2.15) where we replaced $w$ by $u, z$ by $v, z(a, t)$ by $d_{1}(t)$, and $\partial_{x} z(a, t)$ by $d_{2}(t)$. In a same way, by considering formulae similar to (2.17), (2.19), (2.20) and (2.21) where we replace $w$ by $u, z$ by $v, z(b, t)$ by $d_{3}(t)$ and $\partial_{x} z(b, t)$ by $d_{4}(t)$, we determine the quantities

$$
\int_{0}^{T^{*}} u(b, t) \mathrm{d} t \quad \text { and } \quad \int_{0}^{T^{*}} \partial_{x} u(b, t) \mathrm{d} t .
$$

Step 3 . The results from steps 1 and 2 together with the system below determine the parameters $S$ et $\bar{\lambda}$ as follows:

$$
\begin{array}{ll}
L[u](x, t)=\lambda(t) \delta(x-S), & 0<x<\ell, \quad 0<t<T^{*}, \\
L[v](x, t)=R u(x, t), & 0<x<\ell, \quad 0<t<T^{*}, \\
u(0, t)=v(0, t)=0, & 0<t<T^{*}, \\
\partial_{x} u(\ell, t)=\partial_{x} v(\ell, t)=0, & 0<t<T^{*}, \\
u(x, 0)=v(x, 0)=0, & 0<x<\ell .
\end{array}
$$

Multiplying the first equation of (3.1) by $\theta_{i}$, integrating with respect $x$ and $t$ over ]$a, b[\times] 0, T^{*}[$ and by using Green formula, one has

$$
\begin{aligned}
\bar{\lambda} \mathrm{e}^{r_{i} S}=\int_{a}^{b} u\left(x, T^{*}\right) \mathrm{e}^{r_{i} x} \mathrm{~d} x & \\
& +\int_{0}^{T^{*}}\left\{\left[\left(D r_{i}+V\right) \mathrm{e}^{r_{i} x} u(., t)\right]_{a}^{b}-D\left[\mathrm{e}^{r_{i} x} \partial_{x} u(., t)\right]_{a}^{b}\right\} \mathrm{d} t \quad i=1,2 .
\end{aligned}
$$

Therefore, one obtains $S$ and then $\bar{\lambda}$.

Step 4 . A first-kind integral equation for $\lambda(t)$ is derived and a numerical solution algorithm is presented as follows.

First, the solution $(u=u(x, t), v=v(x, t))$ of (3.1) is given by

$$
u(x, t)=\mathrm{e}^{\alpha(S-x)} \sum_{n \geqslant 0} \psi_{n}(S) \psi_{n}(x) \int_{0}^{t} \lambda(\eta) \mathrm{e}^{-\mu_{n}(t-\eta)} \mathrm{d} \eta,
$$

and

$$
v(x, t)=R \mathrm{e}^{-\alpha x} \sum_{n \geqslant 0} \psi_{n}(x) \int_{0}^{t}<\mathrm{e}^{\alpha x} u(., r), \psi_{n}>\mathrm{e}^{-\mu_{n}(t-r)} \mathrm{d} r .
$$




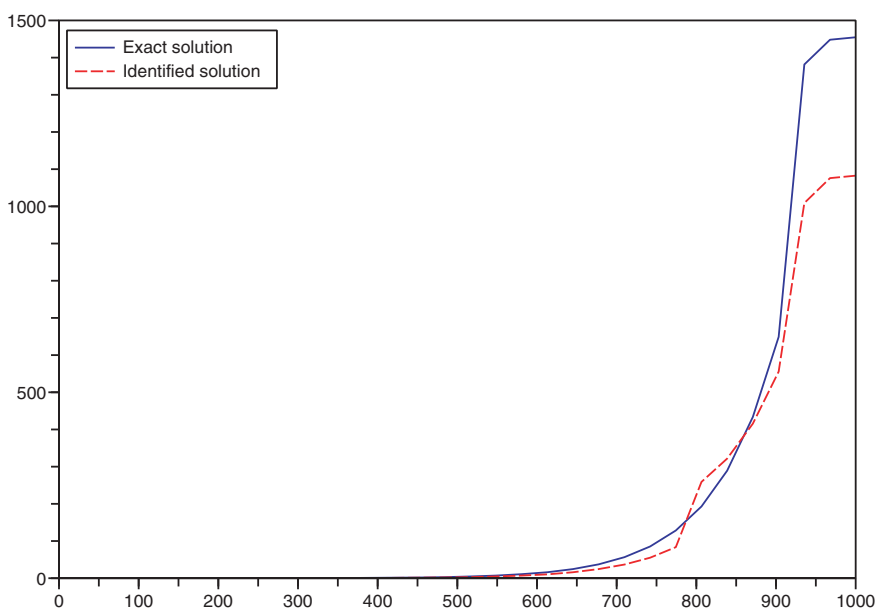

Figure 1. $u_{T}$ obtained from data $d_{1}$ at $a=300 \mathrm{~m}$ with infinite relative error $25.21 \%$.

Then, by substituting (3.3) into (3.4) one obtains

$$
v(x, t)=\int_{0}^{t} \lambda(\eta) \Phi(x, t-\eta) \mathrm{d} \eta
$$

where the function $\Phi$ is given by (2.28).

So the problem that we have to solve here is the following: given $\left\{d_{3}(t), 0<t<T^{*}\right\}$, determine $\lambda$ such that

$$
d_{3}(t)=\int_{0}^{t} \lambda(\eta) \Phi(b, t-\eta) \mathrm{d} \eta, \quad 0<t<T^{*} .
$$

The choice of data $d_{3}(t)$ is justified in section 4 .

Finally, we present below a numerical method for solving the above deconvolution problem. This method consists in replacing the above convolution equation by its approximated version.

Set

$h=\frac{T^{*}}{M}, \quad t_{m}=m h, m=1, \ldots, M, 0<t_{1}<\cdots<t_{m}<\cdots<t_{M-1}<t_{M}$.

and

$$
y_{m}=d_{3}\left(t_{m}\right) \quad \text { and } \quad \lambda^{m}=\lambda\left(t_{m}\right) .
$$

In each interval ] $t_{k}, t_{k+1}\left[\right.$ we approximate the integral $\int_{t_{k}}^{t_{k+1}} \lambda(\zeta) \Phi\left(b, t_{m}-\zeta\right) \mathrm{d} \zeta$ by the trapezoidal rule:

$$
\frac{h}{2}\left(\Phi\left(b, t_{m-k-1}\right) \lambda^{k+1}+\Phi\left(b, t_{m-k}\right) \lambda^{k}\right)
$$

Thus,

$$
\left\{\begin{array}{l}
y_{m}=\frac{h}{2} \sum_{k=0}^{m-1}\left(\Phi\left(b, t_{m-k-1}\right) \lambda^{k+1}+\Phi\left(b, t_{m-k}\right) \lambda^{k}\right) \\
m=1, \ldots, M
\end{array} .\right.
$$

Which leads to a linear system

$$
A \Lambda=Y \text {. }
$$




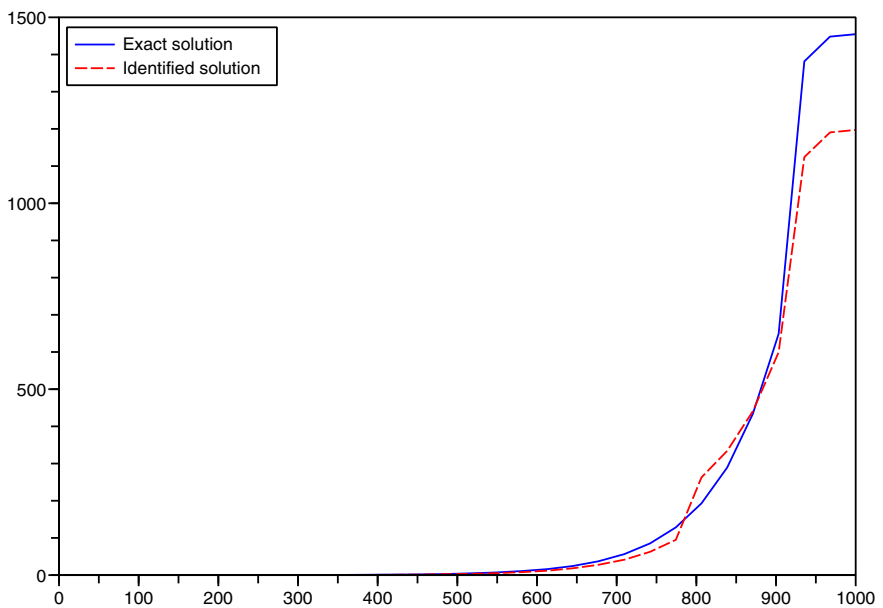

Figure 2. $u_{T}$ obtained from data $d_{3}$ at $a=800 \mathrm{~m}$ with infinite relative error $14.92 \%$.

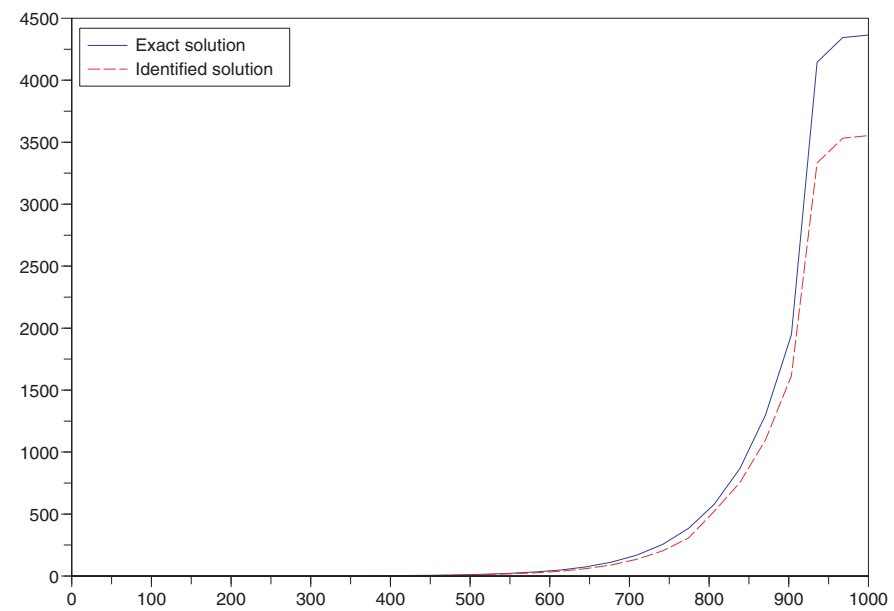

Figure 3. $v_{T^{*}}$ obtained from data $d_{1}$ at $a=300 \mathrm{~m}$ with infinite relative error $16.23 \%$.

\section{Numerical results}

The numerical results are obtained where the length of the considered portion of a river is $\ell=1000 \mathrm{~m}$. Moreover, we suppose observing this portion during a period $T=4 \mathrm{~h}$ and $T^{*}=3 \mathrm{~h}$. In addition, $R=1.01 \times 10^{-5} \mathrm{~s}, V=0.66 \mathrm{~m} \mathrm{~s}^{-1}$ and $D=29 \mathrm{~m}^{2} \mathrm{~s}^{-1}$ [11]. The source is located at $S=600 \mathrm{~m}$ with the intensity

$$
\lambda(t)=\sum_{i=1}^{3} \alpha_{i} \mathrm{e}^{-\beta_{i}\left(t-\tau_{i}\right)^{2}} .
$$

where $\alpha_{1}=1.2, \alpha_{2}=0.4, \alpha_{3}=0.6, \beta_{1}=1 \times 10^{6}, \beta_{2}=5 \times 10^{5}, \beta_{3}=1 \times 10^{6}, \tau_{1}=$ $4500 \mathrm{~s}, \tau_{2}=6500 \mathrm{~s}, \tau_{3}=9000 \mathrm{~s}$.

The purpose of this numerical work is to identify the location $S$ and the intensity $\lambda$ by using the method described in section 4. 


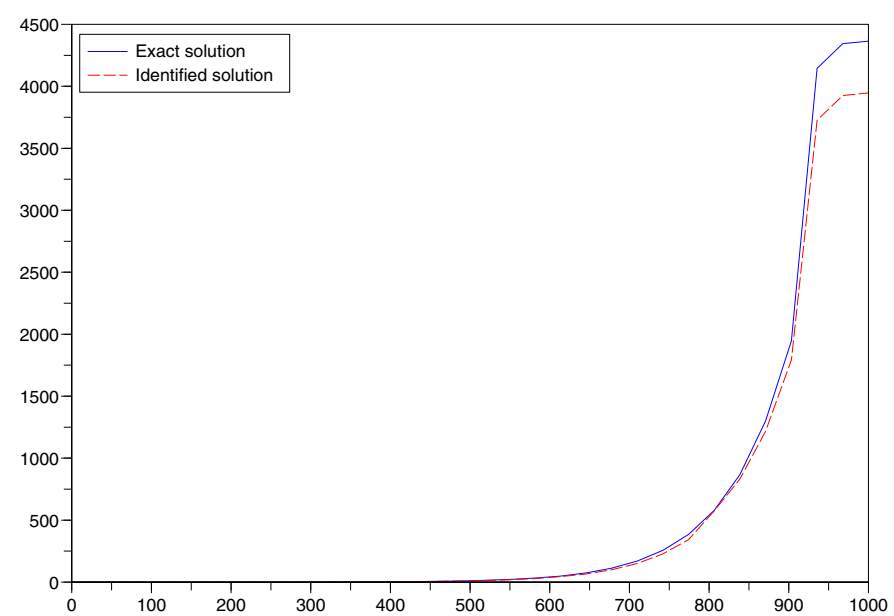

Figure 4. $v_{T} *$ obtained from data $d_{3}$ at $a=800 \mathrm{~m}$ with infinite relative error $9.58 \%$.

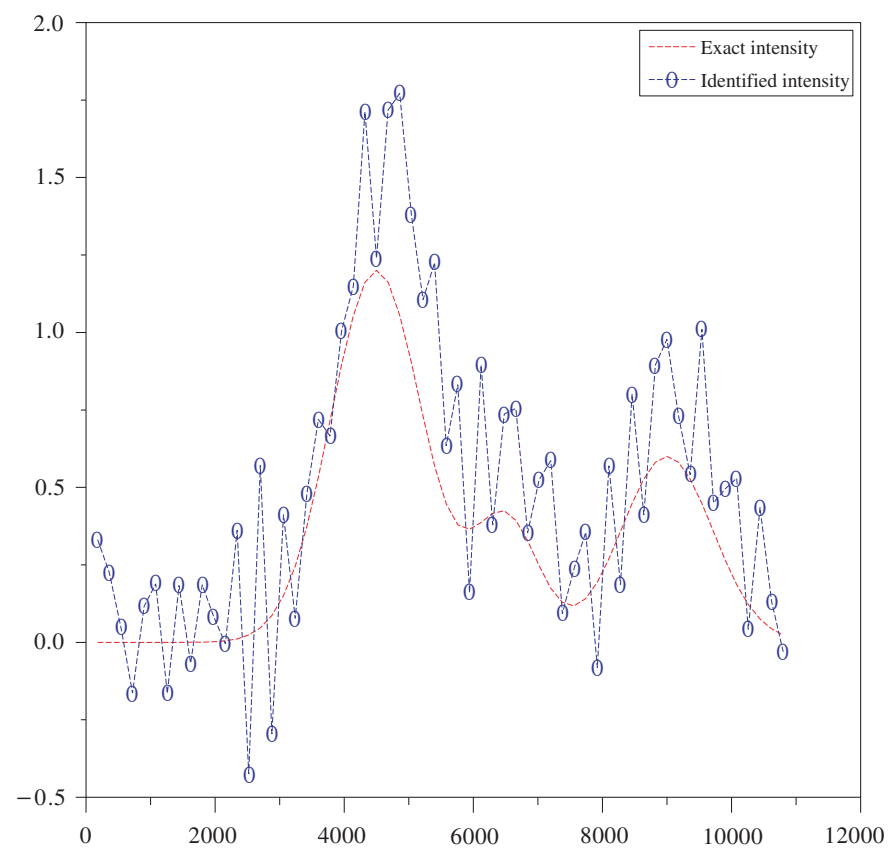

Figure 5. Noise 3\%, with SVD regularization.

The unknown functions $u_{T^{*}}$ and $v_{T^{*}}$ have been determined by using Tikhonov regularization method with a regularization parameter $\varepsilon=0.1$. They are obtained in two ways. The first one by considering the upstream measures $d_{1}(t), T^{*}<t<T$ at $a=300 \mathrm{~m}$. The result concerning $u_{T^{*}}$ is given in figure 1 , while that which concerns $v_{T^{*}}$ is given in figure 3. The second one by considering the upstream measures $d_{3}(t), T^{*}<t<T$ at $a=$ $800 \mathrm{~m}$. The result concerning $u_{T^{*}}$ is given in figure 2, while that which concerns $v_{T^{*}}$ is given in figure 4.

Then, since (3.2), we then obtain $S=576 \mathrm{~m}$. 


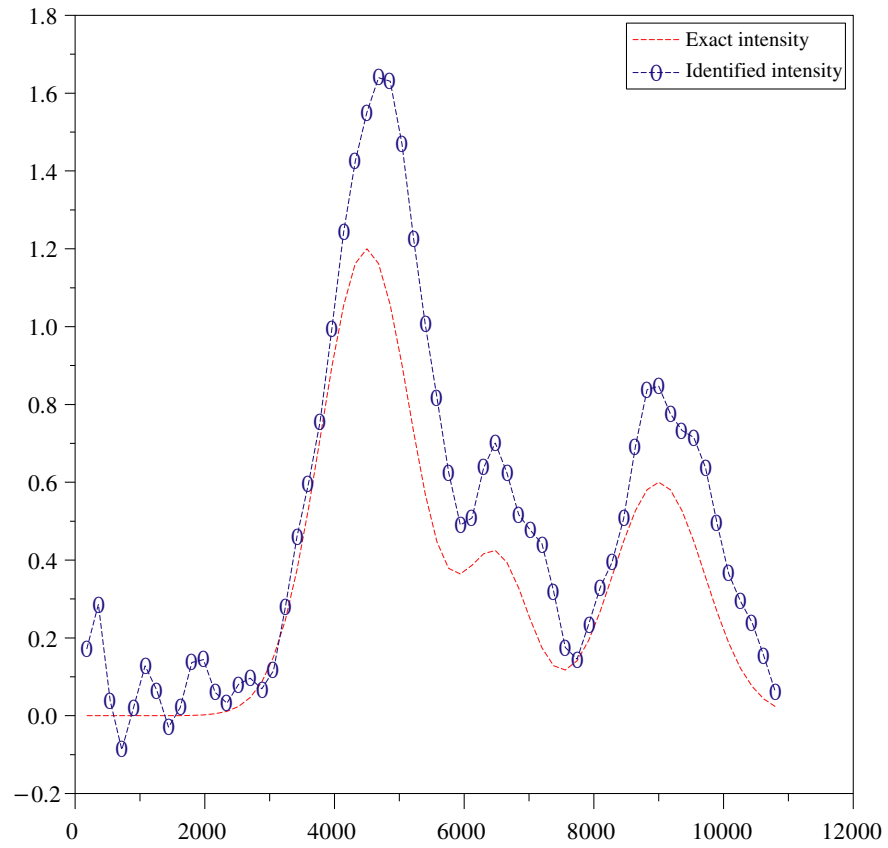

Figure 6. Noise 3\%, with SVD regularization and relative error $46.4 \%$.

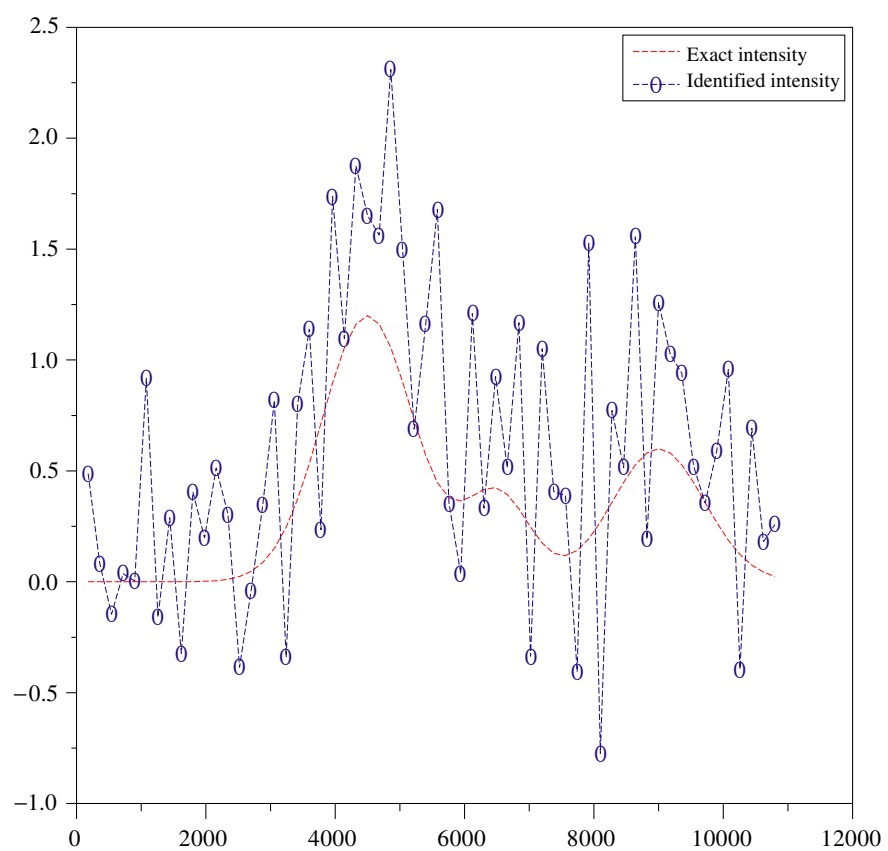

Figure 7. Noise 5\%, without regularization. 


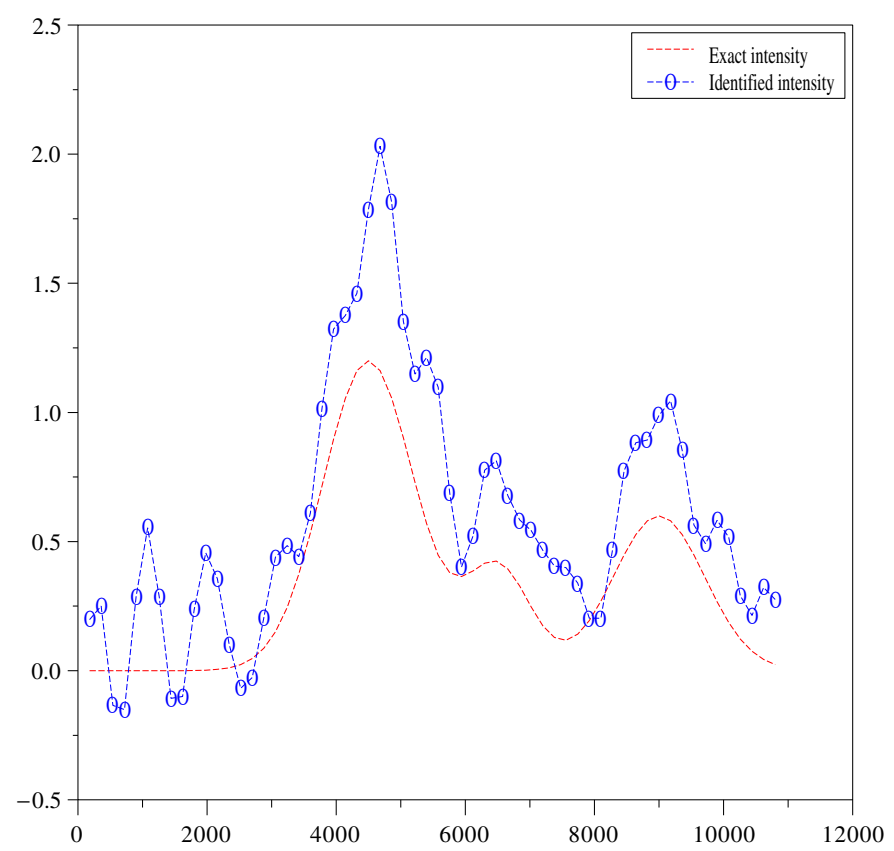

Figure 8. Noise 5\%, with SVD regularization and relative error $72.3 \%$.

Finally, we determine the intensity $\lambda$ by using the above numerical results where figures 5 and 6, respectively figures 7 and 8, compare the exact solution $\lambda$ with that obtained by solving the system (3.5), by using the least-squares method with SVD regularization, with respect to the introduction of Gaussian noise on the data.

\section{Conclusion}

The localization for a point source (location and intensity) for an advection-dispersionreaction system has been studied by two pointwise measurements situated one upstream and the other downstream with respect to the source. Assuming that the source became inactive after some time $T^{*}$, that corresponds, for example, to an accidental pollution stopped at time $T^{*}$, an identifiability result is established, then an identification method of the location is proposed. Finally, the intensity has been determined numerically by a deconvolution method.

Some numerical results are presented. The comparison of these results with those obtained in our previous paper [3] proves that we lose a little precision on the computed localization and the identified intensity function is more sensitive with respect to the introduction of noise on the data, which was foreseeable.

\section{References}

[1] Cannon J R and DuChateau P 1998 Structural identification of an unknown source term in a heat equation Inverse Problems 14 535-51

[2] El Badia A 2005 Inverse source problem in anisotropic medium by boundary measurements Inverse Problems 21 1487-506 
[3] El Badia A, Ha Duong T and Hamdi A 2005 Identification of a point source in a linear advection dispersion reaction equation: application to a pollution source problem Inverse Problems 21 1121-36

[4] El Badia A and Ha Duong T 2002 On an inverse source problem for the heat equation. Application to a pollution detection problem J. Inverse Ill-Posed Problems 10 585-99

[5] Hettlich F and Rundell W 2001 Identification of a discontinuous source in the heat equation Inverse Problems 17 1465-82

[6] Ikehata M 2007 An inverse source problem for the heat equation and the enclosure method Inverse Problems 23 183-202

[7] Linfield C et al 1987 The Enhanced Stream Water Quality Models QUAL2E and QUAL2E-UNCAS: Documentation and User Manual EPA:600/3-87/007 (May)

[8] Ling L, Yamamoto M, Hon Y C and Takeuchi T 2006 Identification of source locations in two-dimensional heat equations Inverse Problems 22 1289-305

[9] Lions J L and Magenes E 1981 Problémes aux limites non homogénes et applications vols 1 and 2 (Paris: Dunod)

[10] Okubo 1980 Diffusion and Ecological Problems: Mathematical Models (New York: Springer)

[11] Roldao J S F, Soares J H P, Wrobel L C, Buge T R and Dias N L C 1991 Pollutant Transport Studies in the Paraiba Do Sul River, Brazil Water Pollution pp 167-80

[12] Titchmarsh E C 1939 Introduction to the Theory of Fourier Integrals (London: Oxford University Press) 